\title{
Delayed control of an elastic beam
}

\author{
Milan Kidd • Gabor Stepan
}

Received: 24 September 2013 / Revised: 27 January 2014 / Accepted: 27 February 2014 / Published online: 21 March 2014

(C) Springer-Verlag Berlin Heidelberg 2014

\begin{abstract}
Stabilization of longitudinal vibrations along an elastic beam with delayed feedback is analyzed. The beam is modeled as a series of equal masses connected by springs and dashpots. The degree of freedom (DoF) of the model is increased-first 1 , then 3 and 9 DoF-with stability charts in terms of the gain and the time delay presented in each case for various levels of damping. With zero damping, for 9 DoF no stability region remains at all. Yet at the continuum case, when the partial delay differential equation is examined with zero damping, discrete stable intervals of gain are found at specific delay values. These intervals largely match the stability charts of the higher DoF models.
\end{abstract}

Keywords Delay $\cdot$ Vibration of continua $\cdot$ Stability

\section{Introduction}

It is a well-known problem of acoustics that the microphoneamplifier-loudspeaker system often generates high-frequency oscillations that lead to inconvenient noise that may destroy the signal to be transmitted and amplified. This problem is a result of the combination of the so-called acoustic echo that refers to cases when the sound arrives back to its source in the air within a few tenths of a second, and the network echo that refers to the same problem when a similar delay is generated electrically in the transmission medium. A good introduction to the combined problem of electroacoustics together with

\footnotetext{
M. Kidd · G. Stepan ( $₫)$

Department of Applied Mechanics, Budapest University

of Technology and Economics, 1521 Budapest, Hungary

e-mail: stepan@mm.bme.hu

M. Kidd

e-mail: kidd.milan@gmail.com
}

several basic methods of the echo cancellation with appropriate filtering can be found in [1,2], while further advanced solutions are presented in recent studies and patents (see, for example, [3-5]). While these methods offer sophisticated solutions, the simplest way to eliminate the problem when this kind of instability occurs in the case of spatially fixed microphones and loudspeakers is to reduce the gain at the amplifier, that is, to reduce the amplification of the signal. This may be, however, just opposite to the original goal of the installation of the system.

Clearly, the reason why it is difficult to predict whether such instabilities occur in the microphone-amplifierloudspeaker system is that time delays are present between the microphone and the loudspeaker in two ways: through the air and also through the control loop via the amplifier. These delays are related to the acoustic echo and the network (or electrically induced) echo, respectively. In this study, a simplified mechanical model of the microphone-amplifierloudspeaker problem is considered. The air is modeled by means of a one dimensional linearly elastic beam with internal viscous damping, and the effect of the time delay in the control loop is also considered at a proportional gain. Thus, the mechanical model presented here is a cantilevered beam with an exciter at the free end creating longitudinal waves and a force measuring device at the fixed end which then feeds back to the exciter with proportional gain $P$ and fixed time delay $\tau$. The end goal is the construction of the stability map in the plane of these two parameters.

A hierarchic construction of the stability charts is presented starting with a 1 degree-of-freedom (DoF) undamped mass and spring model, then steadily higher DoF undamped models are examined in the hope of finding trends that may extend to the continuum model. Next, damping is incorporated into the finite DoF models and trends are noted for increasing DoF and different levels of damping. Finally, 
Fig. $1 n$ DoF mechanical model with delayed $(\tau)$ and proportional $(P)$ feedback

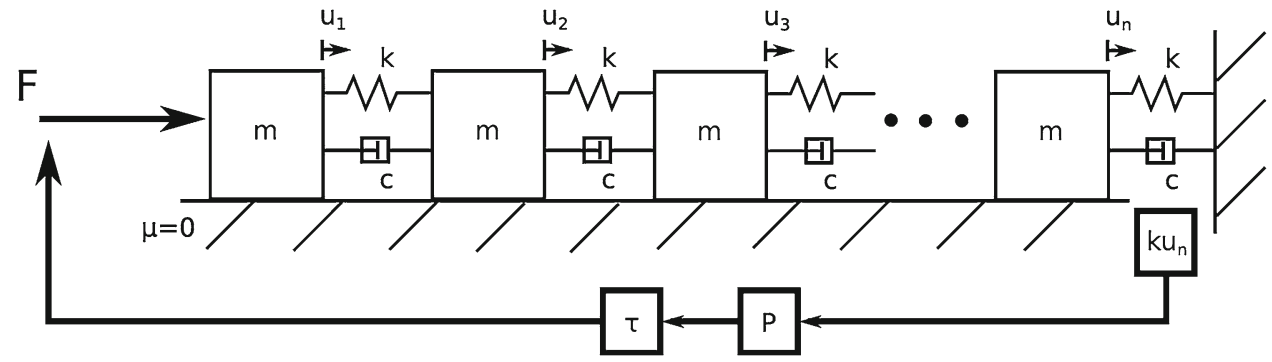

the governing equation-a partial delay differential equation (PDDE) - is constructed for the continuum model with delayed proportional feedback at its boundaries and certain analytically accessible stability results are discussed in comparison with the stability charts of the finite DoF models.

While the studied mechanical model is closely related to an application in electro-acoustics, the underlying mathematical problem is important by itself. The governing PDDE in question can be transformed into a neutral delay differential equation with incommensurate time delays where the so-called $\tau$-stabilizability condition derived in [6] and discussed in details in [7] does not fulfil. While further mathematical studies appeared in this direction recently [8-10], the $\tau$-nonstabilizable case has not been discussed, probably due to the lack of any realistic physical model leading to a system like this. While the goal of this study is still the discussion of the given mechanical model and its linear stability, the underlying mathematical problem requires further investigation along the approach initiated in [11] and continued, e.g., for PDEs in [12] and for DDEs in [13].

\section{Finite DoF mechanical models}

A simplified $n$ DoF model of a continuum beam experiencing longitudinal vibrations is presented in Fig. 1 with general coordinates $u_{j}, j=1, \ldots, n$. The corresponding mass $(m)$, stiffness $(k)$ and damping $(c)$ parameters are chosen to approximate the behavior of the continuum beam having modulus $E$ of elasticity, density $\rho$, cross sectional area $A$ and length $l$ with boundary conditions free-fixed.

In the case of such a beam, the displacement $f$ caused by a static normal load $F$ is expressed by:

$f=\frac{l}{A E} F$.

Equivalently, in the $n$ DoF mechanical model:

$u_{1}=n \frac{F}{k}$.

Thus, $u_{1}=f$ yields the stiffness parameter $k$ :

$k=n \frac{A E}{l}$.
The first natural frequency of the beam is denoted by $\omega_{\mathrm{n}}$ for longitudinal vibrations. Its value can be calculated as found in [14]:

$\omega_{\mathrm{n}}=\frac{\pi}{2 l} \sqrt{\frac{E}{\rho}}$,

and we tune the mass parameter $m$ in a way that the first natural frequency of the discrete model matches this value. This means, for example, that for $n=1$ :

$m=4 \frac{\rho A l}{\pi^{2}}$,

and as $n$ increases it tends to $\rho A l / n$.

The damping is considered to be internal and proportional (Rayleigh type):

$c=\frac{2 \zeta_{1}}{\omega_{\mathrm{n}}} k$,

where $\zeta_{1}$ is the damping ratio describing the first damped vibration mode with a typical value in $[0,1]$. The external damping is considered to be zero, which is represented by the zero coefficient of friction, $\mu$, in Fig. 1.

As explained in the introduction, the feedback loop is based on sensing the spring force $k u_{n}$ at the fixed end of the beam, which is amplified by the constant proportional gain $P$ while discrete time delay $\tau$ also appears in the electric network. The resulting control force $F(t)=P k u_{n}(t-\tau)$ is actuated at the free end of the beam as represented in Fig. 1. We consider that the compressive control forces are far smaller than the critical load of buckling.

Clearly, the governing equation of motion assumes the form:

$\mathbf{M} \ddot{\mathbf{u}}(t)+\mathbf{C} \dot{\mathbf{u}}(t)+\mathbf{K u}(t)+\mathbf{B u}(t-\tau)=\mathbf{0}$,

where $\mathbf{u}=\operatorname{col}\left[u_{1}, \ldots, u_{n}\right]$ and the corresponding matrices are

$\mathbf{M}=m \mathbf{I}, \quad \mathbf{C}=\frac{c}{k} \mathbf{K}$, 


$$
\begin{aligned}
\mathbf{K} & =\left[\begin{array}{ccccc}
k & -k & 0 & \cdots & 0 \\
-k & 2 k & \ddots & \ddots & \vdots \\
0 & \ddots & \ddots & \ddots & 0 \\
\vdots & \ddots & \ddots & \ddots & -k \\
0 & \cdots & 0 & -k & 2 k
\end{array}\right]_{n \times n}, \\
\mathbf{B} & =\left[\begin{array}{llll}
0 & \cdots & -P & k \\
0 & \cdots & 0 \\
\vdots & \ddots & \vdots \\
0 & \cdots & 0
\end{array}\right]_{n \times n},
\end{aligned}
$$

with I denoting the $n \times n$ unit matrix.

\section{Stability analysis of finite DoF models}

In this section, we give a detailed derivation of the formulation of the stability chart for the 1 DoF case and present the results only for 3 and $9 \mathrm{DoF}$. The stability charts are constructed in the plane of the control parameters, gain $P$ and delay $\tau$, while the mechanical and geometrical parameters are considered to be fixed.

\subsection{Stability charts for 1 DoF models}

For the 1 DoF case, those equations of motion (7) and (8) simplify to:

$m \ddot{u}_{1}(t)+c \dot{u}_{1}(t)+k u_{1}(t)-P k u_{1}(t-\tau)=0$,

which can also be presented in the form:

$\ddot{u}_{1}(t)+2 \zeta_{1} \omega_{\mathrm{n}} \dot{u}_{1}(t)+\omega_{\mathrm{n}}^{2} u_{1}(t)-P \omega_{\mathrm{n}}^{2} u_{1}(t-\tau)=0$.

Applying Laplace transformation or substituting the trial solution $u_{1}=e^{\lambda t}$, with the characteristic exponent $\lambda$, the characteristic equation can be obtained:

$D(\lambda):=\lambda^{2}+\omega_{\mathrm{n}}^{2}-P \omega_{\mathrm{n}}^{2} e^{-\lambda \tau}=0$.

From here, the method presented in [15] is used for plotting the D-curves of the stability map. These curves are the borders of regions of a fixed number of unstable characteristic exponents. Thus, precisely at the D-curve, characteristic exponents are crossing the imaginary axis. Therefore, if $\lambda=\mathrm{i} \omega$, then the characteristic equation, separated into its real and imaginary parts, leads to:

$\operatorname{Re} D(\mathrm{i} \omega):-\omega^{2}+\omega_{\mathrm{n}}^{2}-P \omega_{\mathrm{n}}^{2} \cos (\omega \tau)=0$,

$\operatorname{Im} D(\mathrm{i} \omega): P \omega_{\mathrm{n}}^{2} \sin (\omega \tau)=0$.
These equations (13) and (13) can be solved for $P$ and $\tau$, allowing a parametric plot of the D-curves:

$$
\begin{aligned}
P & = \pm \sqrt{\frac{\omega^{4}}{\omega_{\mathrm{n}}^{4}}+2\left(-1+2 \zeta_{1}^{2}\right) \frac{\omega^{2}}{\omega_{\mathrm{n}}^{2}}+1} \\
\tau & =\frac{1}{\omega}\left(\arctan \left(\frac{2 \zeta_{1} \omega \omega_{\mathrm{n}}}{\omega_{\mathrm{n}}^{2}-\omega^{2}}\right)+j \pi\right), \quad j \in \mathbb{N} .
\end{aligned}
$$

In the undamped case, when $\zeta_{1}=0,(14)$ and (15) can be condensed into an analytic equation for the D-curves:

$P=\frac{-(j \pi)^{2}+\omega_{\mathrm{n}}^{2} \tau^{2}}{\omega_{\mathrm{n}}^{2} \tau^{2}}(-1)^{j}, \quad j \in \mathbb{N}$.

To determine the movement of the characteristic exponents at points on the D-curves-whether they cross the imaginary axis from left to right and become unstable or the opposite-requires a partial derivative of the characteristic function $D(\lambda)$ in terms of a parameter, in this case first $P$ and then $\tau$. Only the real part is necessary, as movement of a characteristic exponent $\lambda$ in the imaginary direction has no effect on its stability. Thus, calculated by implicit differentiation:

$$
\begin{aligned}
& \left.\operatorname{Re} \frac{\partial D}{\partial P}\right|_{\lambda=\mathrm{i} \omega} \\
& =\frac{P \omega_{\mathrm{n}}^{4} \tau-2 \omega_{\mathrm{n}}^{2} \omega \sin (\omega \tau)}{\left(P \omega_{\mathrm{n}}^{2} \tau \cos (\omega \tau)\right)^{2}+\left(2 \omega-P \omega_{\mathrm{n}}^{2} \tau \sin (\omega \tau)\right)^{2}}, \\
& \left.\operatorname{Re} \frac{\partial D}{\partial \tau}\right|_{\lambda=\mathrm{i} \omega} \\
& =\frac{-2 P \omega_{\mathrm{n}}^{2} \omega^{2} \cos (\omega \tau)}{\left(P \omega_{\mathrm{n}}^{2} \tau \cos (\omega \tau)\right)^{2}+\left(2 \omega-P \omega_{\mathrm{n}}^{2} \tau \sin (\omega \tau)\right)^{2}} .
\end{aligned}
$$

These partial derivatives at the first few D-curves are presented in Table 1 . Note that the sign of these derivatives is all that's needed, and the denominator in every case is positive. This results in the stability charts plotted with the dimensionless parameters $P$ and $\omega_{\mathrm{n}} \tau$. The first is in Fig. 2 with zero damping, $\zeta_{1}=0$, and the second is in Fig. 3 with $\zeta_{1}=0.01$.

Table 1 The real component of the partial derivatives $\frac{\partial D}{\partial P}$ and $\frac{\partial D}{\partial \tau}$ at the first few D-curves

\begin{tabular}{lll}
\hline$j$ & $\operatorname{Re} \frac{\partial D}{\partial P}$ & $\operatorname{Re} \frac{\partial D}{\partial \tau}$ \\
\hline 0 & $\frac{P \omega_{\mathrm{n}}^{4} \tau}{P^{2} \omega_{\mathrm{n}}^{4} \tau^{2}}$ & 0 \\
1 & $\frac{P \omega_{\mathrm{n}}^{4} \tau^{3}}{P^{2} \omega_{\mathrm{n}}^{4} \tau^{4}+4 \pi^{2}}$ & $\frac{2 P \omega_{\mathrm{n}}^{2} \pi^{2}}{P^{2} \omega_{\mathrm{n}}^{4} \tau^{4}+4 \pi^{2}}$ \\
2 & $\frac{P \omega_{\mathrm{n}}^{4} \tau^{3}}{P^{2} \omega_{\mathrm{n}}^{4} \tau^{4}+16 \pi^{2}}$ & $\frac{-8 P \omega_{\mathrm{n}}^{2} \pi^{2}}{P^{2} \omega_{\mathrm{n}}^{4} \tau^{4}+16 \pi^{2}}$ \\
$\ldots$ & $\cdots$ & $\cdots$ \\
$n$ & $\cdots$ & $\frac{(-1)^{n-1} 2 n^{2} P \omega_{\mathrm{n}}^{2} \pi^{2}}{P^{2} \omega_{\mathrm{n}}^{4} \tau^{4}+(2 n \pi)^{2}}$ \\
\hline
\end{tabular}




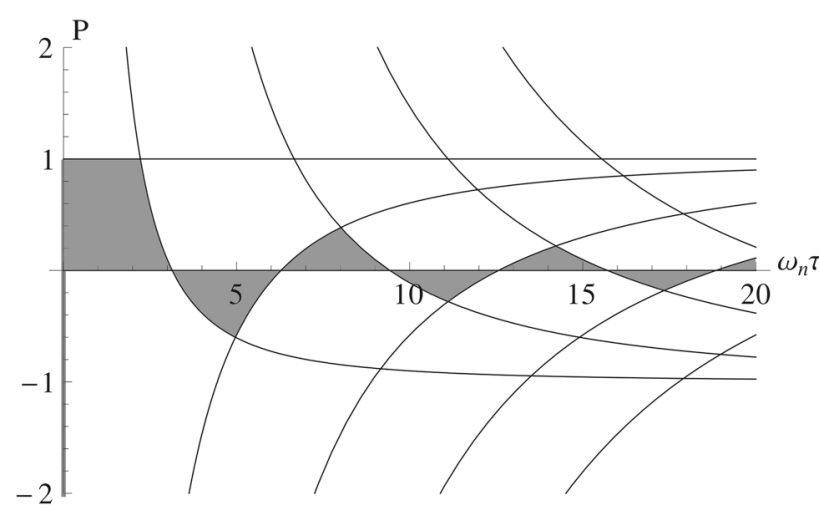

Fig. 2 Stability chart of 1 DoF model with zero damping, $\zeta_{1}=0$. Stable regions are shaded

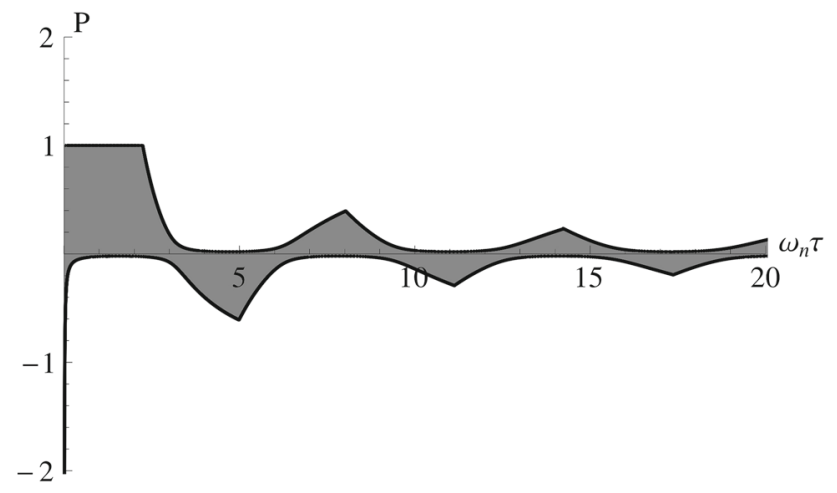

Fig. 3 Stability chart of 1 DoF damped model, $\zeta=0.01$. Stable regions are shaded

\subsection{Stability charts for higher DoF models}

In Figs. 4 and 5 are the stability charts for the 3 DoF model with zero damping, $\zeta_{1}=0$, and with small damping, $\zeta_{1}=$ 0.01 , respectively. In both cases it can be seen that D-curves are cutting into the regions of stability as compared to the 1 DoF counterparts in Figs. 2 and 3, and leaving behind more fragmented regions of stability. For the damped case, this is only so because of the low damping factor. If it were the case that $\zeta_{1}=0.1$, then the $1 \mathrm{DoF}$ and the $3 \mathrm{DoF}$ stability charts would be nearly identical.

Now follow the same charts for the 9 DoF model where a peculiar phenomenon shows up and is inherited by all higher DoF systems, too. In the undamped case, in Fig. 6, there is no region of stability at all apart from the interval $P \in[-1.014,1]$ in the vertical $P$-axis, at $\tau=0$. The Dcurves fully fragmented the remaining stable regions. For the damped case in Fig. 7, there are again regions of stability sliced out as in the $3 \mathrm{DoF}$ case.

Further, in Fig. 8, an even smaller damping ratio is used, $\zeta_{1}=0.001$, cutting away still more of the stable regions, yet sharp peaks remain.

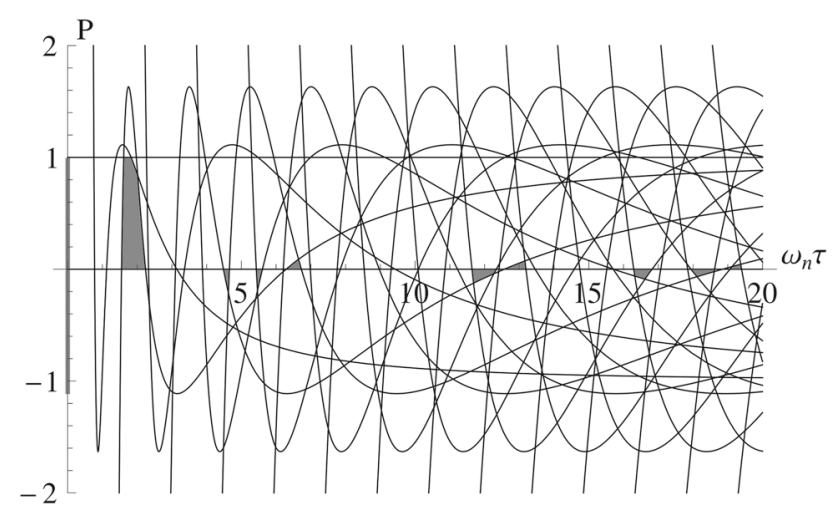

Fig. 4 Stability chart for 3 DoF model with zero damping, $\zeta_{1}=0$. Stable regions are shaded

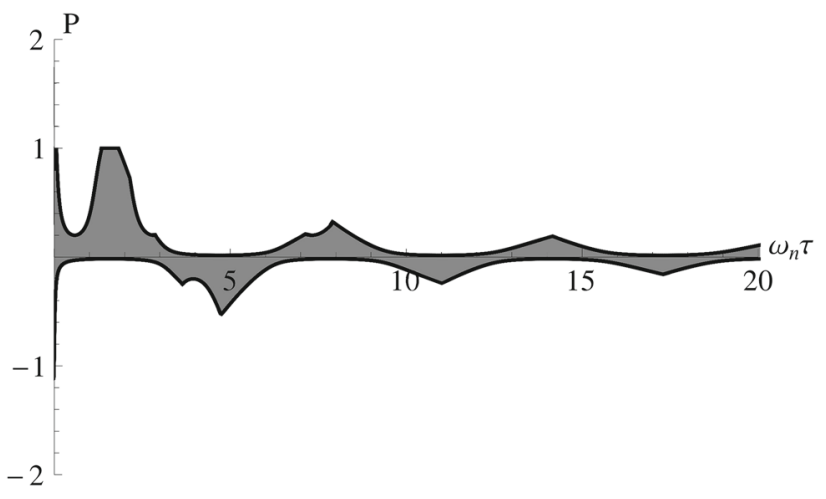

Fig. 5 Stability chart for $3 \mathrm{DoF}$ damped model, $\zeta_{1}=0.01$. Stable regions are shaded

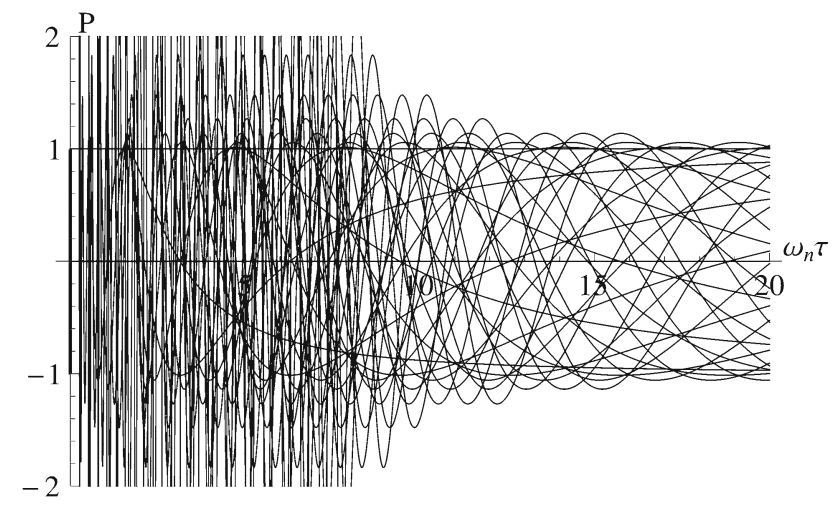

Fig. 6 Stability chart for 9 DoF model with zero damping, $\zeta_{1}=0$. Only the first $30 \mathrm{D}$-curves are plotted-there are no stable regions left among them

On the other hand, if the damping ratio is increased to $\zeta_{1}=0.1$, then again the 1 , the 3 and the 9 DoF stability charts are nearly identical, as shown in Fig. 9.

\section{Stability calculations in the continuum model}

Considering the fragmented structure of the stability charts in case of high DoF models with small damping, it seems to 


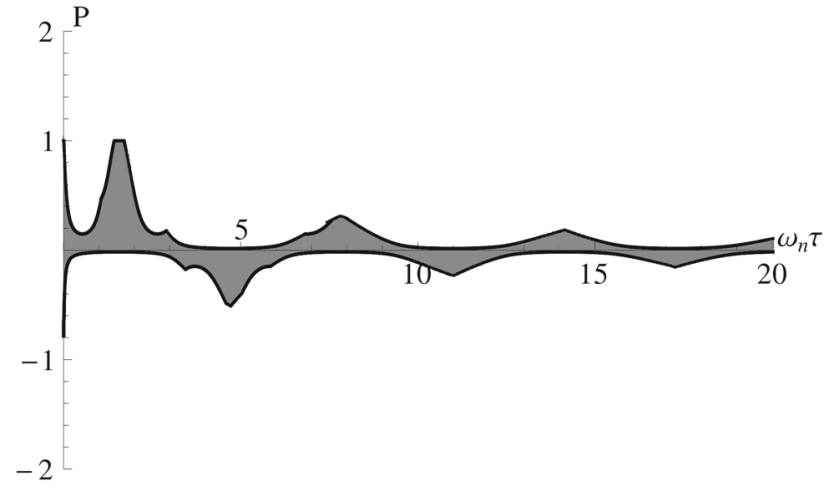

Fig. 7 Stability chart for 9 DoF damped model, $\zeta_{1}=0.01$. Stable regions are shaded

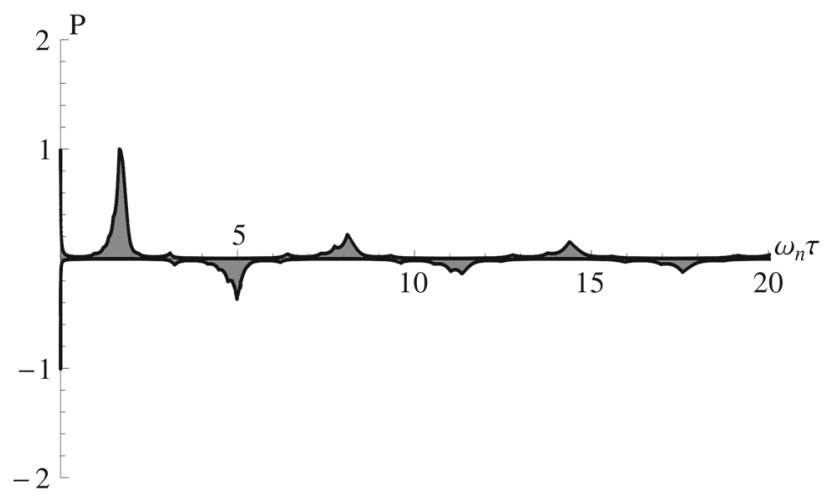

Fig. 8 Stability chart for 9 DoF damped model, $\zeta_{1}=0.001$. Stable regions are shaded

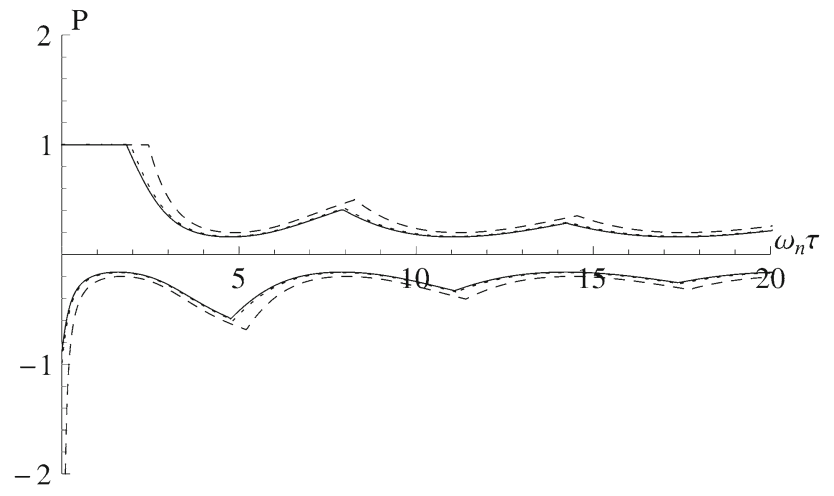

Fig. 9 Stability boundaries for 1 (dashed), 3 (dotted) and 9 (solid) DoF damped model, $\zeta_{1}=0.1$

be useful to compare these results with stability calculations for infinite DoF continuum models with delayed feedback at the boundaries.

\subsection{Construction of PDDE and characteristic equation}

Extending the model from finite to infinite DoF means that the equation of motion changes from an ODE to a partial differential equation (PDE), while the control problem with delay changes from a DDE to a PDDE [16].

As it is known from basic textbooks on vibrations (see for example [14]), the governing equation of the longitudinal vibrations of a homogeneous, prismatic continuum beam assumes the form:

$\ddot{u}(x, t)-c^{2} u^{\prime \prime}(x, t)=0$,

where $u(x, t)$ describes the longitudinal displacement of the cross section of the beam at spatial position $x$ at time $t$. The speed of sound is denoted by $c$, which depends on the modulus $E$ of elasticity and the density $\rho$ :

$c=\sqrt{\frac{E}{\rho}}$.

The boundary conditions correspond to the arrangement shown in Fig. 1. The $x=l$ end, where the longitudinal vibrations are being measured, is fixed:

$u(l, t)=0$.

Furthermore, the vibrations are measured as changes in the normal force $N=A E u^{\prime}$, with prime denoting the derivative with respect to the spatial coordinate $x$. This force is then fed back, with gain $P$ and delay $\tau$, to the force exciter at position $x=0$. This results in:

$P \cdot N(l, t-\tau)=N(0, t)$,

$\Rightarrow P u^{\prime}(l, t-\tau)=u^{\prime}(0, t)$.

Given the PDE (19), the exponential trial solution can be used:

$u(x, t)=U(x) e^{\lambda t}, \quad \lambda \in \mathbb{C}$,

where $U(x)$ is a twice continuously differentiable function satisfying the ODE:

$U^{\prime \prime}(x)-\frac{\lambda^{2}}{c^{2}} U(x)=0$,

with boundary conditions

$U(l)=0$ and $U^{\prime}(0)-P U^{\prime}(l) e^{-\lambda \tau}=0$.

Again, using an exponential trial solution in the form $U(x)=$ $K e^{\psi x}, \psi \in \mathbb{C}$, we obtain:

$\psi_{1,2}= \pm \frac{\lambda}{c}$.

Substituting the general solution

$U(x)=K_{1} e^{\psi_{1} x}+K_{2} e^{\psi_{2} x}$,

into the boundary conditions (25) results in:

$\left[\begin{array}{cc}e^{\frac{\lambda l}{c}} & e^{-\frac{\lambda l}{c}} \\ \frac{\lambda}{c}\left(1-P e^{\frac{\lambda l}{c}-\lambda \tau}\right) & -\frac{\lambda}{c}\left(1-P e^{-\frac{\lambda l}{c}-\lambda \tau}\right)\end{array}\right]\left[\begin{array}{l}K_{1} \\ K_{2}\end{array}\right]=\left[\begin{array}{l}0 \\ 0\end{array}\right]$ 
The determinant of the leading matrix gives the characterstic equation:

$\frac{\lambda}{c}\left(1+e^{-2 T \lambda}-2 P e^{-(T+\tau) \lambda}\right)=0$,

with the substitution of $T$, the time it takes a wave to travel along the length $l$ of the beam:

$T=\frac{l}{c}$.

\subsection{Stability analysis of undamped continuum model}

The stability analysis is carried out in three steps, starting with the uncontrolled case $(P=0)$, then the controlled but undelayed case ( $P>0, \tau=0)$, and finally considering the full PDDE (29) with positive gain $P>0$ and positive delay $\tau>0$.

\subsubsection{Stability analysis of the uncontrolled system}

Beginning with the simplest case, that of zero feedback, $P=$ 0 , the characteristic equation (29) can be expressed as:

$1+e^{-2 T \lambda}=0$

from which all the infinitely many characteristic exponents are pure imaginary:

$\Rightarrow \lambda_{k}=\mathrm{i} \frac{\pi}{2 T}(2 k-1), \quad k \in \mathbb{Z}$.

Thus, there is stability in the linear system, though not asymptotic. From the viewpoint of stability, this result is equivalent to that of the finite DoF models without damping.

\subsubsection{Stability analysis of the controlled undelayed system}

If the system is controlled with gain $P>0$ but there is no time delay in the feedback loop, that is $\tau=0$, the characteristic equation assumes the form:

$1+e^{-2 T \lambda}-2 P e^{-T \lambda}=0$.

Introduce the so-called characteristic multiplier $\mu \in \mathbb{C}$, defined by

$\mu=e^{T \lambda}$,

wherein $\operatorname{Re} \lambda \leq 0 \Leftrightarrow|\mu| \leq 1$. This yields the new form of the characteristic equation:

$\mu^{2}-2 P \mu+1=0$.

The two roots $\mu_{1,2}$ of (35) must satisfy

$\mu_{1} \mu_{2}=1$,

$\mu_{1}+\mu_{2}=2 P$.
Given $|\mu| \leq 1$, (36) is equivalent to $\mu_{1,2}$ both being located on the unit circle. Furthermore, since $\mu_{1}=\bar{\mu}_{2}$, we have:

$\mu_{1}+\mu_{2}=2 \operatorname{Re} \mu_{1}$,

which means that the linear system is stable, though not asymptotically, if and only if

$P \in[-1,1]$.

This exactly matches the trend in the finite DoF models as shown in the vertical $P$-axis of the charts in Figs. 2, 4 and 6 . In the latter case, for the undamped $9 \mathrm{DoF}$ model, the interval of stability on the $P$-axis at $\tau=0$ was $P \in[-1.014,1]$.

\subsubsection{Stability analysis of the controlled system with delay}

Now incorporating feedback, $P>0$, and dropping the $\lambda=0$ trivial root, the characteristic equation (29) assumes the form:

$1+e^{-2 T \lambda}-2 P e^{-(T+\tau) \lambda}=0$,

which corresponds to the extension as $n \rightarrow \infty$ of the undamped finite DoF models examined in Sect. 3. In that case, already for $9 \mathrm{DoF}$ there was no region of stability at all for $\tau>0$. For the continuum case, we can make use of a result from Hale and Verduyn Lunel [6]:

In a scalar difference equation of the form

$x(t)-\sum_{j=1}^{M} a_{j} x\left(t-\tau_{j}\right)=0$,

if the components of $\tau=\left(\tau_{1}, \tau_{2}, \ldots, \tau_{M}\right)$ are rationally independent - that is $\tau_{i} / \tau_{j} \notin \mathbb{Q}$ (the set of rational numbers), $i \neq j$-then the zero solution of (41) is exponentially stable if and only if

$\sum_{j=1}^{M}\left|a_{j}\right|<1$.

Applying this to the characteristic equation (40):

$\sum_{j=1}^{2}\left|a_{j}\right|=|-1|+|2 P|>1 \Leftrightarrow|P|>0$.

So, if the time delays are rationally independent and the feedback is non-zero, the system will be exponentially unstable.

If the time delays, however, are rationally dependent, exponential stability might still be possible. For example, if $\tau=3 T$, then (40) becomes:

$1+e^{-2 T \lambda}-2 P e^{-4 T \lambda}=0$.

Once again, defining a characteristic multiplier by:

$\mu=e^{2 T \lambda}$, 

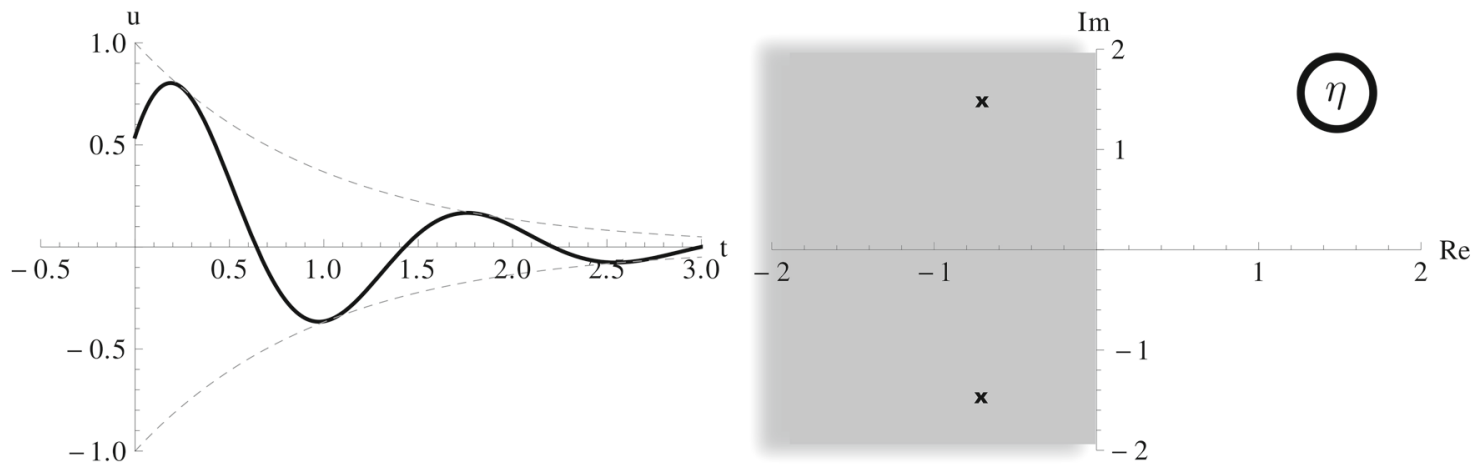

$$
\Downarrow u(x, t)=U(x) e^{\lambda t} \quad \eta=\frac{\mu+1}{\mu-1} \Uparrow \quad \Downarrow \mu=\frac{\eta+1}{\eta-1}
$$

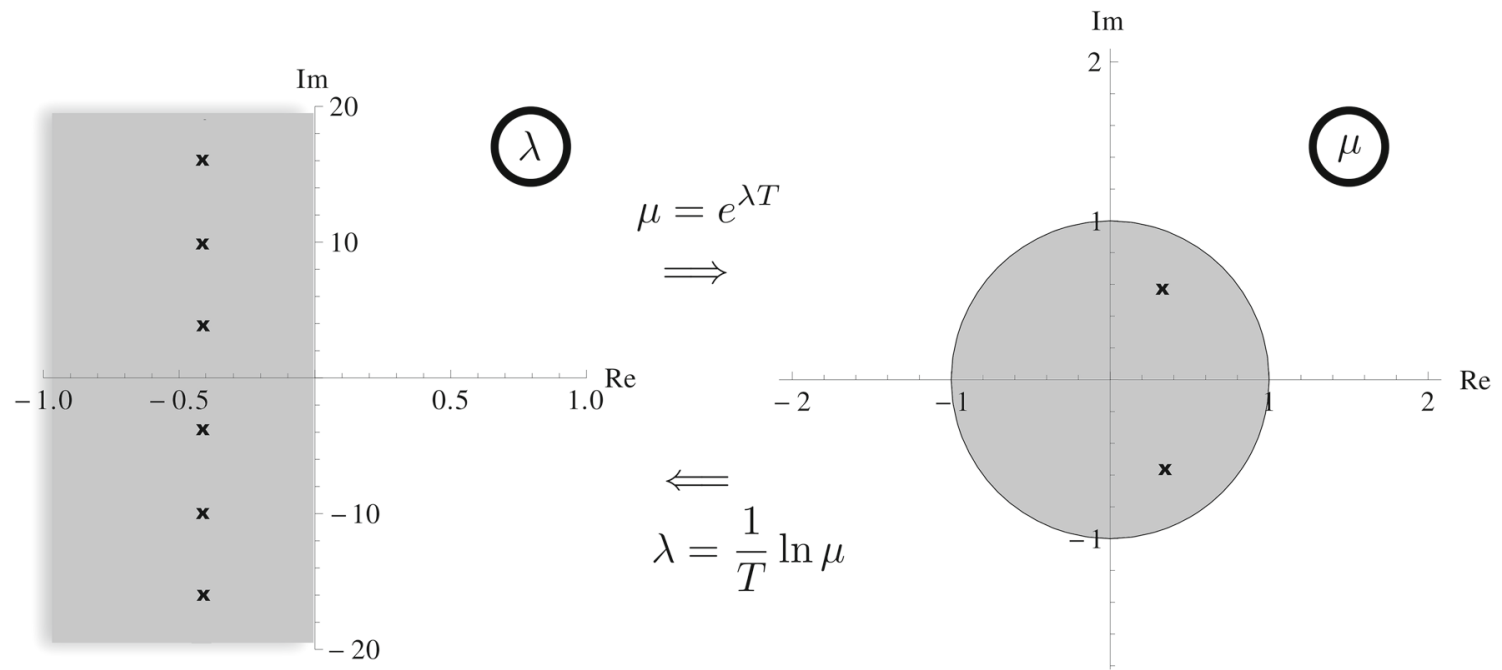

Fig. 10 Transformation of the trial solution to the plane of the characteristic exponent $\lambda$, characteristic multiplier $\mu$ and the Möbius transformation to $\eta$. Stable regions are shaded

and substituting it into (44) we arrive at the characteristic equation:

$\mu^{2}+\mu-2 P=0$.

Following the procedures used also in the cluster treatment of characteristic roots (CTCR) method [17], the Möbius transformation:

$\mu=\frac{\eta+1}{\eta-1}, \quad \eta \in \mathbb{C}$,

is used in order to check $|\mu|<1 \Leftrightarrow \operatorname{Re} \eta<0$. This allows the application of the Routh-Hurwitz Theorem for the polynomial with respect to $\eta$. All the steps of this series of transformations and the stability conditions are represented in Fig. 10. The Möbius transformation leads to:

$(1-P) \eta^{2}+(1+2 P) \eta-P=0$.

For all three coefficients to have the same sign, actually to be positive, and thus the linear system to be exponentially stable, the necessary and sufficient condition is that

$P \in(-1 / 2,0)$.

Other ratios of $\tau$ and $T$ can be tested similarly, but ultimately, only those $\tau$ values that are odd integer multiples of $T$ result in intervals of stability like (49). They are tabulated in Table 2. Analytic solutions are easily accessible until $\tau=5 T$; for larger values we present the approximate numerical values only.

\section{Conclusions and trends}

To compare the result of Sect. 4.2.3-that intervals of stability remain even in the undamped continuum model—with the finite DoF models, first the value of $T$ corresponding to these finite DoF model must be calculated. It follows from (30), (20) and (4) that: 
Table 2 Values of $\tau$ and corresponding stability intervals of $P$

\begin{tabular}{ll}
\hline$\tau=0$ & $P \in[-1,1]$ \\
$\tau=T$ & $P \in(0,1)$ \\
$\tau=3 T$ & $P \in\left(-\frac{1}{2}, 0\right)$ \\
$\tau=5 T$ & $P \in\left(0, \frac{-1+\sqrt{5}}{4}\right)$ \\
$\tau=7 T$ & $P \in(-0.223,0)$ \\
$\tau=9 T$ & $P \in(0,0.174)$ \\
$\tau=11 T$ & $P \in(-0.142,0)$ \\
$\cdots$ & $\cdots$ \\
\hline
\end{tabular}

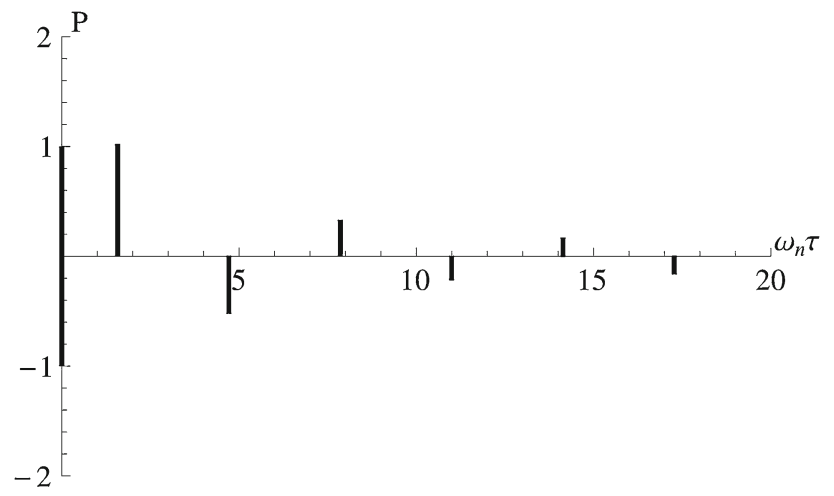

Fig. 11 Stability intervals in $P$ at $\tau=(2 k-1) \pi / 2$

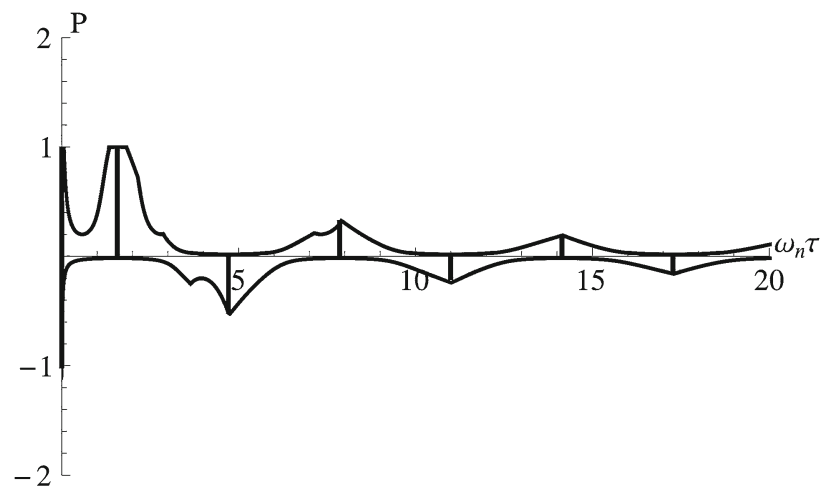

Fig. 12 Stability intervals overlaid atop $3 \mathrm{DoF}$ stability chart for $\zeta_{1}=0.01$

$T=\frac{l}{\sqrt{E / \rho}} \Rightarrow \omega_{\mathrm{n}} T=\frac{\pi}{2}$.

These stability intervals are plotted in Fig. 11.

Comparing these to the moderate damping ratio of $\zeta_{1}=$ 0.01 in the 3 and 9 DoF cases shows relative alignment, as seen in Figs. 12 and 13.

Yet as the damping ratio is decreased and the D-curves of the higher modes cut away more of the stable regions, the alignment does not remain, as seen most drastically in Fig. 14 but also in Fig. 15.

This imperfect alignment is understandable, as it is the higher modes at which the finite DoF model less accurately

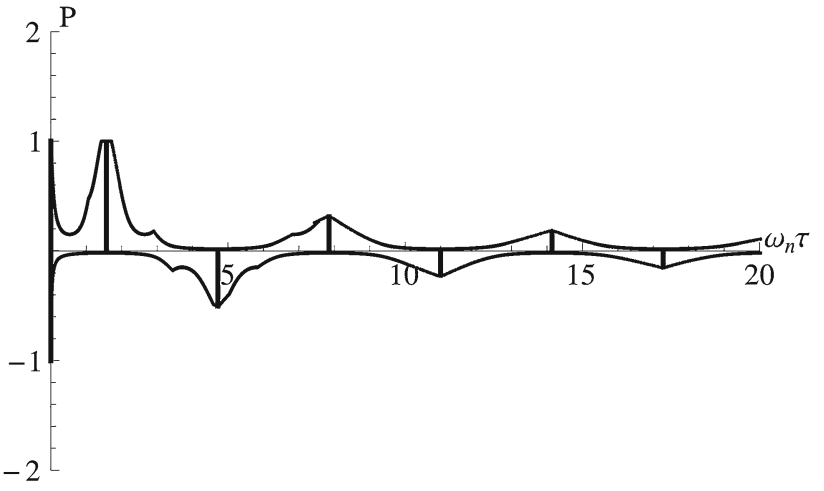

Fig. 13 Stability intervals overlaid atop 9 DoF stability chart for $\zeta_{1}=0.01$

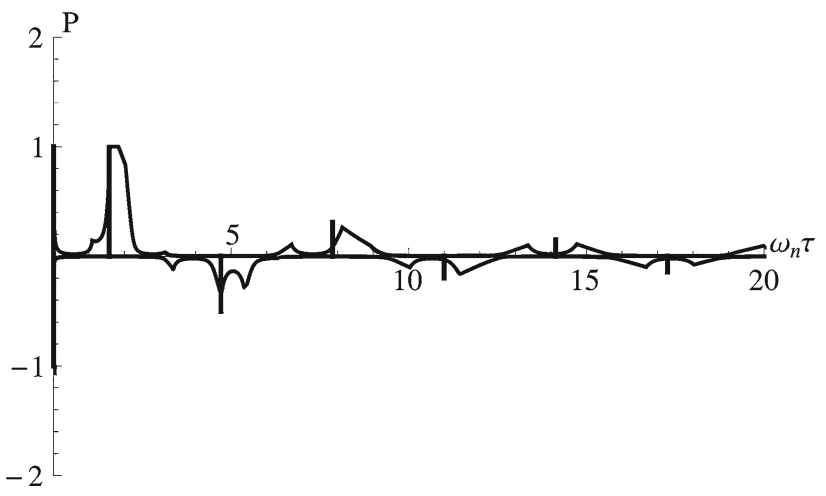

Fig. 14 Stability intervals overlaid atop $3 \mathrm{DoF}$ stability chart for $\zeta_{1}=0.001$

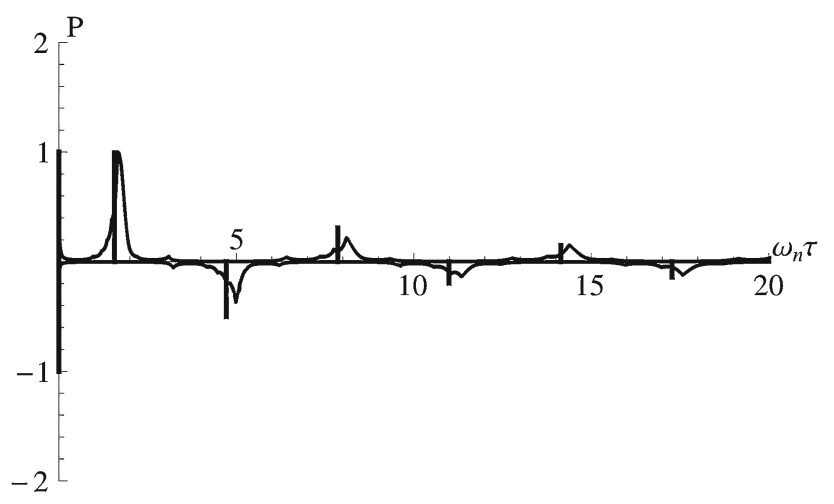

Fig. 15 Stability intervals overlaid atop 9 DoF stability chart for $\zeta_{1}=0.001$

approximates the continuum model, and for smaller damping ratios it is the D-curves of these higher modes that cut away the stability regions. Thus, these removed regions do not align symmetrically relative to the intervals of the continuum model. It could be imagined that for steadily higher DoF models, the alignment would improve even as the damping ratio approached zero.

The other result of the continuum model is that the interval of non-asymptotic stability on the $P$-axis, when $\tau=0$, is 
$P \in[-1,1]$. In the finite DoF models, the upper bound was always 1 , but the lower bound was steadily approaching -1 with increasing DoF. This is further encouragement that differences in the stability charts will only decrease with higher order models.

Acknowledgments This research was supported by the Hungarian Scientific Research Foundation OTKA under Grant No. K101714.

\section{References}

1. Leach WM Jr (2003) Introduction to electroacoustics and audio amplifier design. Kendall/Hunt Publishing Co., Dubuque

2. Benesty J, Gänsler T, Morgan D, Sondhi M, Gay S (2001) Advances in network and acoustic echo cancellation. Springer, Berlin

3. Mader A, Puder H, Schmidt GU (2000) Step-size control for acoustic echo cancellation filters-an overview. Signal Process 80(9):1697-1719

4. Sondhi M, Morgan DR, Hall JL (1995) Stereophonic acoustic echo cancellation-an overview of the fundamental problem. Signal Process Lett IEEE 2(8):148-151

5. Reed M, Hawksford MJ (2010) Acoustic echo cancellation. US Patent 7,660,425, 9 Feb 2010

6. Hale JK, Verduyn Lunel SM (2002) Strong stabilization of neutral functional differential equations. IMA J Math Control Inf 19:5-23

7. Sipahi R, Olgac N (2006) Complete stability analysis of neutraltype first order two-time-delay systems with cross-talking delays. SIAM J Control Optim 45(3):957-971
8. Fabiano RH, Turi J (2007) Preservation of stability in neutral systems. In: Proceedings of 3rd IFAC symposium on system, structure and control (SSSC07), Foz do Iguassu, Brazil, 17-19 October 2007. pp 1-6

9. Michiels W, Niculescu SI (2007) Stability and stabilization of time delay systems - an eigenvalue based approach. SIAM, Philadelphia

10. Sipahi R, Olgac N, Breda D (2010) A stability study on first-order neutral systems with three rationally independent time delays. Int J Syst Sci 41(12):1445-1455

11. Trotter HF (1958) Approximation of semi-groups of operators. Pac J Math 8:887-919

12. Ito K, Kappel F (1998) The Trotter-Kato theorem and approximation of PDEs. Math Comput 67:21-44

13. Ito K, Turi J (1991) Numerical methods for a class of singular integro-differential equations based on semigroup approximation. SIAM J Numer Anal 28:1698-1722

14. de Silva CW (1999) Vibration: fundamentals and practice, 1st edn. CRC, Boca Raton

15. Stepan G, Insperger T (2011) Semi-discretization for time-delay systems. Springer, New York

16. Wu J (1996) Theory and application of partial functional differential equations. Springer, New York

17. Olgac N, Sipahi R (2005) The cluster treatment of characteristic roots and the neutral type time-delayed systems. ASME J Dyn Syst Meas Control 127:88-97 